\title{
Exercise Effects on Risk of Cardiovascular Disease among Iranian Women
}

\author{
* Corresponding Author; \\ Address: Health Education \\ Department, Tarbiat Modares \\ University, P.O. Box 14115-331, \\ Tehran, Iran \\ E-mail: aminsh_f@modares.ac.ir
}

Received: Oct 11, 2010

Accepted: Jan 11, 2011

Key Words: Exercise; Running; Cardiovascular Disease; Risk Factor; Screening; Framingham Risk Score; Blood Pressure; Women

\author{
Farkhondeh Amin-Shokravi*1, PhD; Reza Rajabi ${ }^{2}$, PhD; Nargess Ziaee ${ }^{2,3}$, MSc
}

\begin{abstract}
Purpose: Physical inactivity is more prevalent among women than men, varies by ethnic group, and becomes increasingly prevalent with age. The aim of this study was to examine the effects of a 12-week exercise program on the cardiovascular disease risk and fitness of Iranian middle aged women.

Methods: This was a randomized controlled trial study. Participants in the training group $(n=20)$ performed treadmill running exercise at a high intensity $(70-80 \%$ of maximum heart rate, $0 \%$ grade) for 30 min/day, 3 days/week. On the other hand, participants in the control group $(n=20)$ were asked to maintain their habitual lifestyle and not change their activity or dietary habits. Measurements of body mass index, waist/hip ratio, blood pressure, total cholesterol, and lipoprotein subtractions were taken before program and after 12 weeks. Changes in 10-year risk scores for coronary heart disease were calculated using Framingham risk equation.
\end{abstract}

Results: Significant decreases in systolic and diastolic blood pressure, reduction in 10-year risk of coronary heart disease, and reduction in lipid levels were found within the training group between baseline and 12-week measurements. No changes were found in these parameters within the control group.

Conclusions: The study provides evidence for the positive effects of exercise training on the reduction of cardiovascular disease risks among women aged 40-55 years.

Asian Journal of Sports Medicine, Volume 2 (Number 1), March 2011, Pages: 37-43

\section{INTRODUCTION}

C oronary heart disease (CHD) is one of the most important leading causes of premature disability and death among women worldwide ${ }^{[1,2,3]}$. Over the last decade, there has been a high rate of risk factors among women, particularly among obese women with health problems such as physical inactivity, diabetes mellitus, hypertension, smoking and high plasma cholesterol levels ${ }^{[3]}$. Physical inactivity is more prevalent among women than men $^{[3]}$.

Participation in regular and low to moderate intensive physical activity for $30 \mathrm{~min} /$ day, 3 days/week has been related to reduced risk of $\mathrm{CHD}^{[3-8]}$. Since 
many evidences showed that inactive individuals are at higher risk of CHD, avoidance of sedentary life style has been recommended ${ }^{[9,10]}$. It has been verified that physical activity could decrease blood pressure, increase fitness, and improve lipid profiles ${ }^{[2,9,11,12]}$. Besides, it is recommended that adults could have physical activity in a continuous or intermittent fashion (minimum of 10-min bouts) for 20-60 min, 3-5 days per week. However, there are few trials including intervention of suitable intensity and supervised programs $^{[12-15]}$.

There are many studies that predict CHD risk in men and women ${ }^{[9,16-25]}$. CHD risk prediction equations ${ }^{[9]}$ can be applied to estimate the likelihood of an individual developing $\mathrm{CHD}$ in a specific time period. Tully et al ${ }^{[9]}$ studied the effects of 30-min brisk walking, 5 days per week, on health and fitness of individuals aged 50-65 years. According to this study, a significant decrease in 10-year risk of CHD, systolic and diastolic blood pressure and reduction in stroke risk was observed, whereas no significant change in lipid levels or anthropometric measurements were found in any of two groups. The present study was conducted to assess the effects of a treadmill running exercise at a high intensity [70-80\% of maximum heart rate $\left(\mathrm{HR}_{\max }\right), 0 \%$ grade] for $30 \mathrm{~min} /$ day, 3 days/week.

\section{METHODS AND SUBJECTS}

This study was a randomized controlled trial. Eligible participants were recruited from four fitness centers located in different geographically regions of Tehran in 2009. Data were collected at the time of randomization (baseline) and 12-week follow up. All adult women who aged between 45 and 55 years and were referred to these centers were considered eligible if they agreed to take part in the study. Our inclusion criteria were as follows: (1) coronary heart disease, (2) peripheral vascular disease, (3) musculo-skeletal disease, (4) pulmonary disease, (5) diabetes mellitus, or (6) severe uncontrolled hypertension, resting blood pressure $>140 / 90 \mathrm{~mm} \mathrm{Hg}$, body mass index $(\mathrm{BMI})>35$ $\mathrm{kg} / \mathrm{m}^{2}$, currently prescribing lipid lowering medication, postmenopausal female (no menstrual period in the last 12 months), taking hormone replacement therapy in the previous 3 months and being physically active. Individuals with BMI $>35 \mathrm{~kg} / \mathrm{m}^{2}$ were excluded because they were abnormal according to the study performed by Tully et al while our investigation was conducted only in normal women ${ }^{[9]}$ who had no medical problems and did not take any medications in order to prevent any potential bias.

Being physically active was defined as being engaged in more than 30 min of physical activity. Written informed consent was obtained from all subjects following explanation of the research procedures and the probable risks. Adequate compliance was defined as the completion of a 12week treadmill running program (30 min, 3 days/week, $70-80 \% \mathrm{HR}_{\max }, 0 \%$ grade).

In this study, 40 eligible participants were randomly divided into two groups: control group $(\mathrm{N}=20)$ who did not receive any intervention and they were just asked to maintain their habitual lifestyle and not change their activity or dietary habits, and intervention group $(\mathrm{N}=20)$ who received exercise program. Because of the nature of intervention and the length of the study, the full blindness of researcher and participants and who administered the intervention were impossible throughout the study. The statistical analyst was blinded to group assignment. Ethic principals were considered in this study. The participants required to comply with the study protocol. Hence, the purposes and procedures of the study were completely explained to them first and if they signed written consent form, they were entered into the study. This right was kept for participants to withdraw throughout the study. The ethics committee of Tehran University of Medical sciences approved the study.

\section{Intervention:}

The intervention was considered as treadmill running exercise in this study. This exercise program involved treadmill running program determined as $70-80 \%$ $\mathrm{HR}_{\max }, 0 \%$ grade for $30 \mathrm{~min} /$ day, 3 days/week, for 12 weeks. Each session of exercise included 15 min warm up with stretching and running movements followed by continues running with intensity of $70-80 \% \mathrm{HR}_{\max }$. Time of running on treadmill in the first session was 15 
min, and increased each 2 sessions up to 30 minutes. Cool down process was performed at the end of each session for 10 minutes. Before starting training program, maximum heart rate was calculated with Karvonen (220-age) and 70-80\% $\quad \mathrm{HR}_{\max }$ was determined with a SD of 10 to $12^{[2]}$. Exercise intensity was controlled using the pulse belt.

In this study, treadmill exercise program was the independent variable. Besides, BMI, blood pressure, blood lipid and CHD risk estimation was considered as primary outcome or dependent variables.

\section{Measurements:}

Height and body mass were determined using a stadiometer and scales (Seca 709, Germany) respectively. BMI was calculated by diving body mass (kg) by height squared (m2). Waist circumference was taken midway between the ribs and iliac crest or at the smallest natural waistline, and the average of two measures was recorded to the nearest $0.1 \mathrm{~cm}$. Waist-tohip ratio (WHR) was assessed using a fiberglass tape and standard technique ${ }^{[18]}$.

\section{Blood pressure:}

Resting systolic and diastolic blood pressure were recorded as the mean of duplicate measurements, taken at least 2 min apart, using a random zero sphygmomanometer (Acuson MK2, Mountain View, CA) following 20 minutes of quiet sitting.

\section{Blood sampling and lipid analysis:}

Following a 12-h fast, a 10-ml blood sample was taken by venipuncture in a seated position. Blood samples were collected in tubes treated with anticoagulant drug (Ethylen Diamine Teta Acetic Acid=EDTA) or serum clot activator, and then were separated and stored at $70^{\circ} \mathrm{C}$ until utilization. Whole blood was analyzed immediately after collection for hemoglobin and hematocrit to monitor changes in plasma volume. To consider the relevant validity and reliability of the data, the same test and equipments were applied to assess blood pressure, blood lipid levels, total cholesterol (TC), high density lipoprotein cholesterol (HDL-C) and Framingham risk score. Female subjects were also asked to complete a menstrual phase diary to ensure that baseline and post-training blood samples were taken at the same phase of the menstrual cycle. Posttraining blood samples were taken on day 3 after the last exercise session. TC and HDL-C levels were measured from serum samples using enzymatic colorimetric techniques.

\section{CHD risk estimation:}

CHD risk factor score was estimated by applying the Framingham risk score equation ${ }^{[18,20,22-26]}$. This uses an individual's gender, age, blood pressure, smoking status, TC, HDL-C, and diabetic status to calculate a percentage likelihood of a coronary event over a 10year period ${ }^{[7,23,25-29]}$. Some other studies ${ }^{[29-32]}$ used CHD risk factors estimation as a predictor of healthy life.

\section{Ethical permission:}

Committee on the ethics of Research on human beings of Tehran University examined the application and agreed that it covered the objection raised by the committee.

\section{Statistical analysis:}

Data were analyzed using SPSS 14. Differences between control and training groups in blood pressure, blood lipids, and CHD risk score were assessed at baseline and at 12 weeks and the extent of change was compared between the two groups using an independent t-test. Differences within a group (intragroup) were assessed by comparing the results at baseline with those after 12 weeks using a paired t-test. Statistical significance was established at $P<0.05$.

\section{RESULTS}

There were no differences in baseline characteristics between training and control groups (Table 1). Significantly decreased systolic and diastolic blood pressure, 10-year risk of CHD and blood lipid levels, TC, and an increased level of HDL-C were found within the training group between baseline and 12week measurements (Table 2). No significant changes were found in control group. The results of this study showed that mean 
Table 1: Baseline characteristics of participants

\begin{tabular}{lccc} 
Variable & $\begin{array}{c}\text { Training group } \\
\text { Mean (SD) }\end{array}$ & $\begin{array}{c}\text { Control group } \\
\text { Mean (SD) }\end{array}$ & P. value \\
\hline Age (years) & $46.25(1.61)$ & $46.00(1.55)$ & $>0.05$ \\
Height (m) & $1.62(0.04)$ & $1.60(0.03)$ & $>0.05$ \\
Weight (kg) & $72.65(7.88)$ & $70.33(8.12)$ & $>0.05$ \\
Body mass index (kg/m $\mathbf{2}^{2}$ & $27.48(2.02)$ & $27.26(3.41)$ & $>0.05$ \\
Waist-to-Hip ratio & $0.81(0.06)$ & $0.82(0.07)$ & $>0.05$ \\
Systolic blood pressure (mm Hg) & $118.50(4.61)$ & $118.33(4.49)$ & $>0.05$ \\
Diastolic blood pressure (mm Hg) & $76.07(4.93)$ & $77(4.92)$ & $>0.05$ \\
Total cholesterol (mg/dl) & $208.65(29.97)$ & $214.60(34.26)$ & $>0.05$ \\
HDL-C (mg/dl) & $52.60(10.67)$ & $47.20(9.56)$ & $>0.05$ \\
10-year risk estimate for CHD (\%) & 3.7 & 4.0 & $>0.05$ \\
HDL-C: high density lipoprotein cholesterol / CHD: Coronary heart disease &
\end{tabular}

difference of two time measurements between two groups were significant (Table 3). Furthermore, this study demonstrated that a notable reduction in the Framingham risk score was observed across low (5.8\%), moderate (4\%), and high (3.6\%) fitness levels $(P=0.009)$.

\section{DISCUSSION}

The purpose of this study was to evaluate the effectiveness of 30-min supervised, 3 days per week exercise on cardiovascular risk factors (blood pressure, BMI, and cholesterol), Framingham risk score, and fitness in women aged between 40 and 55 years. The hypothesis of the investigation was that the active women with the mentioned amount of regular and low to moderate intensive physical activity are exposed to reduced risk of CHD and stroke and in total, health problems. It has also been proven that exercise as a lifestyle modification can be recommended for all patients with hypertension. The reason is that even small reductions in blood pressure are associated, in long-term, with a decreased risk of cardiovascular disease $^{[32-34]}$. It has been mentioned that diastolic blood pressure is a more potent cardiovascular risk factor than systolic blood pressure until the age of 50, whereas systolic blood pressure is more important thereafter. Therefore, the most cases of hypertensionrelated cardiovascular disease occur at present in the

Table 2: Comparison of mean outcome measurements between training and control groups at 12-week follow up

\begin{tabular}{lccc} 
Variable & $\begin{array}{c}\text { Training group } \\
\text { Mean (SD) }\end{array}$ & $\begin{array}{c}\text { Control group } \\
\text { Mean (SD) }\end{array}$ & P. value \\
\hline Systolic blood pressure (mm Hg) & $115(5.7)$ & $120(5.7)$ & 0.001 \\
Diastolic blood pressure (mm Hg) & $71.1(7.6)$ & $76.3(4.8)$ & 0.002 \\
Total cholesterol (mg/d) & $189.2(24.2)$ & $216.3(24.7)$ & 0.003 \\
HDL-C (mg/d) & $62.2(12.7)$ & $48.4(8.6)$ & 0.001 \\
10-year risk estimate for CHD (\%) & $2.2(1.1)$ & $4.3(1.9)$ & 0.001 \\
HDL-C: high density lipoprotein cholesterol / CHD: Coronary heart disease & &
\end{tabular}


Table 3: The results of paired t-test in case group before and after the intervention

\begin{tabular}{lcccc} 
Variables & Mean (SD) & T & Df & P.value \\
\hline CHD risk score & $0.01 \pm .01$ & 6.5 & 17 & 0.001 \\
Systolic blood pressure (mm Hg) & $3.5 \pm 6.5$ & 2.4 & 19 & 0.002 \\
Diastolic blood pressure (mm Hg) & $5.57 \pm 8.23$ & 3.02 & 19 & 0.007 \\
HDL-C (mg/dl) & $-9.22 \pm 9.21$ & -4.24 & 17 & 0.001 \\
Total cholesterol (mg/dl) & $19.83 \pm 13.66$ & 6.15 & 17 & 0.001
\end{tabular}

HDL-C: high density lipoprotein cholesterol / CHD: Coronary heart disease

middle-aged and elderly ${ }^{[35]}$.

Considering this point in this study, women aged from 40 to 55 years were sampled. The structure of this study has been based on the point that the participation of non-physicians in detecting risk factors for chronic diseases with high morbidity and mortality could be a part of a new health care model for disease prevention, that is possible by applying Framingham equation, in which most of intensive physical activities are in a better physical and mental health status ${ }^{[11,32,35]}$.

It is also demonstrated that three short bouts (10 min) of brisk walking $\left(70 \% \mathrm{VO}_{2 \max }\right)$ accumulated during day are at least as effective as one continuous bout of equal total duration in reducing cardiovascular risk and improving aspects of mood in previously sedentary individuals ${ }^{[2]}$.

In addition, the findings of this study showed that applying the current physical activity recommendations for prevention and health benefit into a 12-week supervised, treadmill running program could decrease cardiovascular risk. This was reflected by decreased systolic and diastolic blood pressure and blood lipid levels (TC) and increased HDL cholesterol and improved Framingham risk score in sedentary adults aged between 40 and 55 years.

These observations could be added to the evidence of the health benefits of moderately intensive exercise to sedentary adults. Significant decreases in systolic and diastolic blood pressure, reduction in 10-year risk of CHD, and reduction in lipid levels were found within the training group between baseline and 12week measurements. No changes were found in these parameters within the control group.
The results of this study suggested that to acquire the full advantages of physical activity, it should be ensured that the activity is carried out in a mode and at an intensity that yields best benefits. For instance, 30min treadmill exercise, $70-80 \% \mathrm{HR}_{\max }$, 3 days/week clearly promotes health and decreases the risk of cardiovascular disease. This is reflected by decreased systolic and diastolic blood pressure and blood lipid levels (TC) and increased HDL-C and reduction of CHD in women with 40-55 years of age.

Some of the results of the present study about the reduction of blood pressure and risk of CHD is in agreement with the results of Bittner, Tully, Murphy and La Monte ${ }^{[3,9,11,20]}$ and in some cases (increasing HDL-C and TC reduction) is contrary to the findings of Tully $^{[9]}$.

Numerous Studies have shown that aerobic physical activity decreases blood lipid levels (TC) ${ }^{[2,5,7,8]}$. The treadmill exercise program done by the participants in this study is an aerobic physical activity and the necessary energy for this activity comes from lipids metabolism or might be a consequence of post physical activity metabolism. Decreased levels of TC and increased levels of HDL-C might be because of these issues as well.

Murphy et $\mathrm{al}^{[2]}$ stated that increased HDL-C could be associated with severe exercise activities and the results of the current study concurs with their results. On the contrary, Tully (in the cases of HDL-C and TC) suggested that this may related to their study protocol ${ }^{[0]}$ which might not be adequate. The severity of applied physical activities in those studies was not sufficient to bring about changes to blood lipids. Meanwhile, 
increased level of serum lipids is a part of HDL risk factors. Therefore, increasing physical activity might reduce risk for CHD.

The results of this study showed that applying the current physical activity recommendations for prevention and health benefit into a 12-week supervised, treadmill running program could decrease cardiovascular risk. However, applying different clinical techniques and equipment to assess study variables and also the role of stress on the rapid changes of these variables can be important limitations of the present study. To prevent the first mentioned bias the same test and equipment were applied to assess blood pressure and blood lipid levels (TC), HDL-C and Framingham risk score. Unfortunately, stress could not be managed.

\section{CONCLUSION}

According to the results of the present study, treadmill exercise program done by the participants could decrease levels of blood lipids such as TC and increase density of HDL-C. Furthermore, it could reduce systolic blood pressure and could consequently lead to a reduction in Framingham risk score.

\section{ACKNOWLEDGMENTS}

Hereby, we would like to appreciate all the participants because this study could not be performed without their kind help.

Conflict of interests: None.

\section{REFERENCES}

1. Stamfer MJ, HU FB, Manson JE, et al. Primary prevention of coronary heart disease in women through diet and lifestyle. $N$ Engl J med 2002;343:16-22.

2. Murphy M, Neville A, Neville C, et al. Accumulating brisk walking for fitness, cardiovascular risk, and psychological health. Med Sci Sports Exerc 2002;34:1468-74.

3. Bittner V. Women and coronary heart disease risk factors. J Cardiovascular Risk 2002:9:315-22.

4. Asikainen TM, Miilunpalo S, Oja P, et al. Randomized, controlled walking trials in postmenopausal women: the minimum dose to improve aerobic fitness? Br J Sports Med 2002;36:189-94.

5. D'amore S, Mora S. Gender-specific prediction of cardiac disease: importance of risk factors and exercise variables. Cardiol Rev 2006;14:281-5.

6. Hu G, Tuomilehto J, Borodulin K, Jousilahti P. The joint associations of occupational, commuting, and leisure-time physical activity, and the Framingham risk score on the 10-year risk of coronary heart disease. Euro Heart J 2007;28:492-8.

7. Sigal RJ, Kenny GP, Wasserman DH, Castaneda -Sceppa C. Physical activity /exercise and type 2 diabetes. Diabetes Care 2004;27:2518-39.

8. Kozakova M, Palombo C, Mhamdi L, et al. Habitual physical activity and vascular aging in a young to middle-age population at low cardiovascular risk stroke. RISC Investigators 2007;38:2549-55.

9. Tully MA, Chan WS, Mc Glade K, Young IS. Brisk walking, fitness, and cardiovascular risk: A randomized controlled trial in primary care. Prev Med 2005;41:622-8.

10. Sawatzky N. Physical activity and cardiovascular health in aging women: A health promotion perspective. J Aging Phys Act 2002;10:396-412.

11. Murphy MH, Neville AM, Murtagh EM, Holder RL. The effect of walking on fitness, fatness and resting blood pressure: A meta-analysis of randomized, controlled trials. Prev Med. 2007;44:377-85.

12. Duncan JJ, Gordon NF, Scott CB. Women walking for health and fitness. How much is enough? JAMA 1991;266:3295-9.

13. Murtagh EM, Boreham CA, Nevill A, et al. The effects of 60 minutes of brisk walking per week, accumulated in two different patterns, on cardiovascular risk. Prev Med 2005;41:92-7.

14. Lee IM; Rexrode KM; Cook NR; et al. Physical Activity and Coronary Heart Disease in Women: is "no pain, no gain" passé? JAMA 2001:285:1447-54. 
15. Gohlke H, Winter M, Karoff M, Held K. CARRISMA: a new tool to improve risk stratification and guidance of patients in cardiovascular risk management in primary prevention. Euro J Cardiovascular Prev Rehabil. 2007;14:141-8.

16. Anderson KM, Odell PM, Wilson PW, Kennel WB. Framingham and Boston, Mass. Cardiovascular disease risk profiles. Am Heart J 1990;121:293-8.

17. Eichler K, Puhan MA, Steurer J, Lucas M. Prediction of first coronary events with the Framingham score: A systematic review. Am Heart J 2007;153:211-8.

18. Lloyd-Jones DM, Wilson PW, Larson MG, et al. Framingham risk score and prediction of lifetime risk for coronary heart disease. Am J Cardio 2004;94:20-4.

19. Wilson PW, D'Agostino RB, Levy D, et al. Prediction of coronary heart disease using risk factor categories. Circulation 1998;97;1837-47.

20. La Monte MJ, Durstine JL, Addy CL, et al. Physical activity, physical fitness, and Framingham 10-year risk score: the cross cultural activity participation study. J Cardiopulm Rehabil 2001;21:63-70.

21. American College of Sports Medicine. ACSM`s Health-related Physical Fitness Assessment Manual. Philadelphia: Lippincott, Williams and Wilkins. 2004; Pp:129-36.

22. Schisterman Ef, Whitcomb BW. Coronary age as a risk factor in modified Framingham risk score. BMC Med Imaging 2004;4:1.

23. Liu J, Hong Y, D’Agostino RB, et al. Predictive value for the chinese population of the framingham chd risk assessment tool compared with the chinese multi-provincial cohort study. JAMA 2004;219:22.

24. Brindle P, Emberson J, Lampe F, et al. Predictive accuracy of the Framingham coronary risk score in British men: prospective cohort study. BMJ 2003;3:27-29.

25. Agostino RB, Grundy S, Sullivan LM, Wilson P. Validation of the Framingham coronary heart disease prediction scores for the CHD Risk prediction group. American Medical Association. JAMA 2001;286-92.

26. Ramachandran S, French JM, Vander p, et al. Using the Framingham model to predict heart disease in the United Kingdom: retrospective study. BMJ 2000;320;676-87.

27. Schisterman EF, Whitcomb BW. Coronary age as a risk factor in modified Framingham risk score. BMC Med Imaging 2004;4:1.

28. Sheridan S, Pig none M, Muldrow C. Framingham-based tools to calculate the global risk of coronary heart disease. A systematic review of tools for clinicians. J Gen Intern Med 2003;18:1039-52.

29. Wilson PW. Physical activity, fitness, and coronary risk estimation. J Cardiopulm Rehabil 2001;21:71-2.

30. Amin-Shokravi F, Alhani F, Kazemnejad A, Vahdaninia M. The relationship between brisk walking with physical and mental health in women. Behbood 2010;14:177-81. (Persian)

31. Amin-shokravi F, Alhani F, Kazemnejad A, Vahdaninia M. The relationship between brisk walking with women quality of life. Payesh 2009;8:407-13. (Persian)

32. Avenell A, Broom J, Brown TJ, et al. Systematic review of the longtermeffects and economic consequences of treatments for obesity and implications for health improvements. Health Technol Assess 2004;8:1-182.

33. Whitworth JA. World Health Organization (WHO)/ International Society of Hypertension (ISH) statement on management of hypertension. J Hypertens 2003;21:1983-92.

34. Vasheghani Farahani A, Mansournia M, Asheri H, et al. The effects of a 10-week water aerobic exercise on the resting blood pressure in patients with essential hypertension. Asian J Sports Med 2010;1:159-67.

35. Franklin SS, Larson MG, Khan SA, et al. Does the relation of blood pressure to coronary heart disease risk change with aging? The Framingham Heart Study. Circulation 2001;103:1245-9. 